\title{
Hydrodynamic Attractors in Phase Space
}

\author{
Michal P. Hellerø, ${ }^{1,2, *}$ Ro Jefferson, ${ }^{1, \dagger}$ Michał Spaliński, ${ }^{2,3, *}$ and Viktor Svensson $\circledast^{2,1, \S}$ \\ ${ }^{1}$ Max Planck Institute for Gravitational Physics, Potsdam-Golm 14476, Germany \\ ${ }^{2}$ National Centre for Nuclear Research, 02-093 Warsaw, Poland \\ ${ }^{3}$ Physics Department, University of Biatystok, 15-245 Biatystok, Poland
}

(Received 27 March 2020; revised 3 July 2020; accepted 27 August 2020; published 22 September 2020)

\begin{abstract}
Hydrodynamic attractors have recently gained prominence in the context of early stages of ultrarelativistic heavy-ion collisions at the RHIC and LHC. We critically examine the existing ideas on this subject from a phase space point of view. In this picture the hydrodynamic attractor can be seen as a special case of the more general phenomenon of dynamical dimensionality reduction of phase space regions. We quantify this using principal component analysis. Furthermore, we adapt the well known slow-roll approximation to this setting. These techniques generalize easily to higher dimensional phase spaces, which we illustrate by a preliminary analysis of a dataset describing the evolution of a five-dimensional manifold of initial conditions immersed in a 16-dimensional representation of the phase space of the Boltzmann kinetic equation in the relaxation time approximation.
\end{abstract}

DOI: 10.1103/PhysRevLett.125.132301

Introduction.-The physics of strong interactions studied in heavy-ion collisions at the RHIC and LHC (see, e.g., Ref. [1] for a concise contemporary review) has been a remarkable source of inspiration for the study of complex systems far from equilibrium. The phenomenological success of relativistic hydrodynamics, together with calculations in microscopic models based on holography and kinetic theory, have inspired several novel research directions. One such direction is centered on the notion of hydrodynamic attractors. These were introduced in Ref. [2] with the aim of capturing universal features of nonequilibrium physics beyond the limitations of the gradient expansion and were subsequently explored in many works, including Refs. [3-32].

In the context of reproducing the spectra of soft particles in ultrarelativistic heavy-ion collisions, the underlying observable of interest is the expectation value of the energy-momentum tensor $\left\langle T^{\mu \nu}\right\rangle$. Ideally, one would like to have a way of predicting its behavior as a function of time directly in QCD. Such calculations remain beyond reach, but have been pursued in quantum field theories possessing a gravity dual [33-35] in a number of works, see Refs. [36,37] for a review. Another line of development replaces QCD by its effective kinetic theory description [38], as reviewed in Refs. [37,39]. The evolution of $\left\langle T^{\mu \nu}\right\rangle$ depends on the initial state, captured by the bulk metric in

Published by the American Physical Society under the terms of the Creative Commons Attribution 4.0 International license. Further distribution of this work must maintain attribution to the author(s) and the published article's title, journal citation, and DOI. Funded by SCOAP . holography, or by the initial distribution function in the kinetic theory. After some time, the vast majority of this information is effectively lost, and $\left\langle T^{\mu \nu}\right\rangle$ evolves hydrodynamically. These explorations have led to the idea of a hydrodynamic attractor, identified in Ref. [2] in a class of hydrodynamic theories [40-42] as a specific solution which is approached by generic solutions initialized at arbitrarily small times. It was also observed there that a "slow-roll" condition akin to what is used in inflationary cosmology $[43,44]$ leads to an accurate approximation of this attractor. Subsequent works have lead to many interesting phenomenological applications $[2,5,16,45,46]$.

Despite these developments, there are three important yet largely unexplored issues. The first addresses the different concepts of attractor, the second concerns their relevance for the dynamics of initial states of interest and the third is the question of their existence beyond highly symmetric settings. We address these points with the goal of clearing the ground for new developments, in particular for generalizations to more realistic flows with less symmetry. Our approach is based on the phase space picture, i.e., the space of variables needed to parametrize the dynamics underlying $\left\langle T^{\mu \nu}\right\rangle$, which was introduced in this context in Refs. [11,47].

Dissipation of initial state information.-There are two key features of the dynamics following an ultrarelativistic heavy-ion collision: the expansion that drives the system away from equilibrium and interactions that favor equilibration [13]. The simplest model of this is Bjorken flow which assumes one-dimensional expansion and boost-invariance along the expansion axis, conveniently described in terms of proper time $\tau$ and spacetime rapidity $y$. In interacting conformal theories, at asymptotically late 
times the system follows a scaling solution for the (effective) temperature [48]

$$
T(\tau)=\frac{\Lambda}{(\Lambda \tau)^{1 / 3}}+\cdots
$$

In this equation, the dimensionful constant $\Lambda$ is the only trace of initial conditions. Corrections to this come in two forms: higher-order power-law terms which are sensitive only to $\Lambda$, and exponential corrections which encode information about the initial conditions and describe its dissipation $[2,4,12,49-51]$. This asymptotic form is usually referred to as a transseries [52]. The simplest models where this can be observed explicitly are formulated in the language of hydrodynamics.

Models of hydrodynamics. - We primarily focus on hydrodynamic theories (see Ref. [53] for a review), which despite their name include transient nonhydrodynamic excitations needed to avoid acausality. In such models, $\left\langle T^{\mu \nu}\right\rangle$ is decomposed into a perfect fluid term and a "dissipative" part denoted by $\pi^{\mu \nu}$ :

$$
\left\langle T^{\mu \nu}\right\rangle=(\mathcal{E}+\mathcal{P}) u^{\mu} u^{\nu}+\mathcal{P} g^{\mu \nu}+\pi^{\mu \nu},
$$

where $u_{\mu} u^{\mu}=-1, u_{\mu} \pi^{\mu \nu}=0$, and the energy density $\mathcal{E}$ and pressure $\mathcal{P}$ are related by the thermodynamic equation of state. In the conformal case considered here, $\mathcal{P}=\mathcal{E} / 3$. Conservation equations of $\left\langle T^{\mu \nu}\right\rangle$ provide the four equations of motion for $\mathcal{E}$ and $u^{\mu}$. Hydrodynamic models, building on the original ideas of Refs. [40,41], provide the remaining equations for $\pi^{\mu \nu}$ in terms of relaxation-type dynamics that ensure matching to the hydrodynamic gradient expansion of any microscopic model.

In this Letter, we consider two classes of models. The first one is the Müller-Israel-Stewart (MIS) model $[40,41]$

$$
\tau_{\pi} \mathcal{D} \pi^{\mu \nu}=-\pi^{\mu \nu}+\eta \sigma^{\mu \nu},
$$

where $\mathcal{D} \pi^{\mu \nu} \equiv u^{\alpha} \nabla_{\alpha} \pi^{\mu \nu}+\ldots$, is the Weyl-covariant derivative in the comoving direction $[54,55]$ and $\left.\sigma^{\mu \nu}=2 \mathcal{D}^{(\mu} u^{\nu}\right)$ is the shear tensor. One can supplement Eq. (3) with additional terms defining the so-called Baier-RomatschkeSon-Starinets-Stephanov (BRSSS) model [42] and in the following we will refer to it as MIS-BRSSS models. Conformal symmetry requires that

$$
\eta=C_{\eta} \frac{\mathcal{E}+\mathcal{P}}{T} \quad \text { and } \quad \tau_{\pi}=\frac{C_{\tau_{\pi}}}{T} \quad \text { with } \quad \mathcal{E} \sim T^{4},
$$

where $C_{\eta}, C_{\tau_{\pi}}$ are dimensionless constants and $T$ is defined as the temperature of an equilibrium state at the same energy density $\mathcal{E}$. Equation (3) implies relaxation phenomena on a time scale defined by the relaxation time $\tau_{\pi}$.

For the Bjorken flow, the combined equations reduce to a second order ordinary differential equation (ODE) for the effective temperature $T(\tau)$, see Eq. (7.17) in Ref. [53].
The hydrodynamic attractor was originally observed in this model in Ref. [2] using a special scale-invariant parametrization involving pressure anisotropy (note $\pi_{2}^{2}=\pi_{3}^{3}$ )

$$
\mathcal{A}=\frac{\pi_{2}^{2}-\pi_{y}^{y}}{\mathcal{P}}=6+18 \tau \frac{\dot{T}(\tau)}{T(\tau)},
$$

which is understood as a function of time measured by

$$
w=\tau T(\tau) .
$$

We denote derivatives with respect to $\tau$ with a dot and derivatives with respect to $w$ with a prime. In contrast with $T(\tau), \mathcal{A}(w)$ satisfies a first order ODE, see Eq. (7.18) in Ref. [53].

The second hydrodynamic theory of interest here is the Heller-Janik-Spalinski-Witaszczyk (HJSW) model [55] (see also Ref. [56]), in which Eq. (3) is replaced by

$$
\left\{\left(\frac{1}{T} \mathcal{D}\right)^{2}+\frac{2}{T^{2} \tau_{\pi}} \mathcal{D}\right\} \pi^{\mu \nu}=-\frac{\tau_{\pi}^{-2}+\omega^{2}}{T^{2}}\left\{\pi^{\mu \nu}+\eta \sigma^{\mu \nu}\right\} .
$$

This structure again ensures relaxation phenomena on a timescale $\tau_{\pi}$, but here they occur in an oscillatory manner as in $\mathcal{N}=4$ supersymmetric Yang-Mills theory [57], with frequency $\omega=C_{\omega} T$. This model leads to a third order ODE for the effective temperature $T(\tau)$ or a second order ODE for $\mathcal{A}(w)$, see Eq. (7.26) in Ref. [53], and provides a workable setting with a richer set of initial conditions than offered by MIS-BRSSS models.

Hydrodynamic attractors. - The hydrodynamic attractor in MIS-BRSSS models arose through studying a range of solutions for $\mathcal{A}(w)$ that converge and then evolve toward local thermal equilibrium [2]. This behavior is known in the mathematical literature [58] as a forward attractor. Intuitively, forward attractors are solutions that attract nearby sets as $w \rightarrow \infty$. In MIS-BRSSS models, every solution is a forward attractor. Conversely, by considering solutions initialized at earlier and earlier times we observe that generic solutions (which diverge at $w=0$ ) decay to a specific solution which is regular there. Such behavior is known as a pullback attractor.

These two notions of attractors, introduced in this context in [47] are concerned with different regimes: the forward attractor describes asymptotically late times while the pullback attractor is a statement about early times. In MIS-BRSSS models, there is a unique solution which is both a pullback attractor and a forward attractor; we denote this solution by $\mathcal{A}_{\star}(w)$.

Attractors and phase space.-The above discussion raises two concerns. The first is that were one to visualize a range of solutions $T(\tau)$, the hydrodynamic attractor would not be apparent, and yet it is encoded there since $\mathcal{A}(w)$ can be clearly derived from $T(\tau)$. If a different observable showed attractor behavior how would one find 
it? This issue must be clarified if one aims to identify attractors in more complicated settings. We address it by considering the full phase space parametrized by $(\tau, T, \dot{T})$ for MIS-BRSSS models and $(\tau, T, \dot{T}, \ddot{T})$ for the HJSW model. We include proper time as one of the phase space variables because we are dealing with a nonautonomous system.

The second concern stems from the fact that forward and pullback attractors strongly depend on either asymptotically late or asymptotically early time dynamics. Such asymptotic regimes may be inaccessible or unphysical. We address this by focusing on the local dynamics on families of constant- $\tau$ slices of phase space, on which solutions of the equations of motion appear as points (see Figs. 1 and 3). However, any particular solution $\mathcal{A}(w)$ corresponds to a different curve on each slice, as dictated by Eq. (5). On the basis of earlier studies, one expects that, as $\tau$ increases, different solutions (points on constant- $\tau$ slices) will collapse to the curves representing the attractor $\mathcal{A}_{*}(w)$. This is what one eventually sees in Figs. 1 (bottom) and 3 (bottom-right), but numerical studies of phase space histories reveal a much finer picture. The process of information loss occurs in three phases: local dimensionality reduction, approach to the hydrodynamic attractor loci (red curves in Fig. 1), and evolution toward equilibrium along the attractor. Consider a finite "cloud" of initial states, such as any one of the three colored sets of points shown in Fig. 1. Each cloud contracts and becomes one dimensional. When this happens depends on the initial conditions. For example, the brown cloud in Fig. 1 (the one initialized at smallest value of $\tau_{0} T$ ) loses a dimension quite early and rather far from the attractor, while the other two do this much later.

Quantifying dimensionality reduction.-We have argued that dimensionality reduction in phase space is an important feature of hydrodynamic attractors, but so far we have not provided a working recipe to quantify this process. A promising direction, which we only begin to explore here, follows from recognizing that dimensionality reduction is one of the basic tasks of machine learning. For the simple cases considered here, principal component analysis (PCA) is quite effective (see Refs. [59-63] for other applications of PCA to problems in ultrarelativistic heavy-ion collisions). Intuitively, PCA quantifies the variations of a data set in different directions and associates an explained variance with each of them.

We start by applying PCA to the two-dimensional phase space of MIS-BRSSS models. On the initial time slice we pick a state $(T, \dot{T})$ and consider a random set of points within a disc around it. For this set of points, the two principal components are approximately equal in magnitude. At each time step we recompute the principal components for the set of states we started with (see Fig. 1) and their evolution in time is shown in Fig. 2.
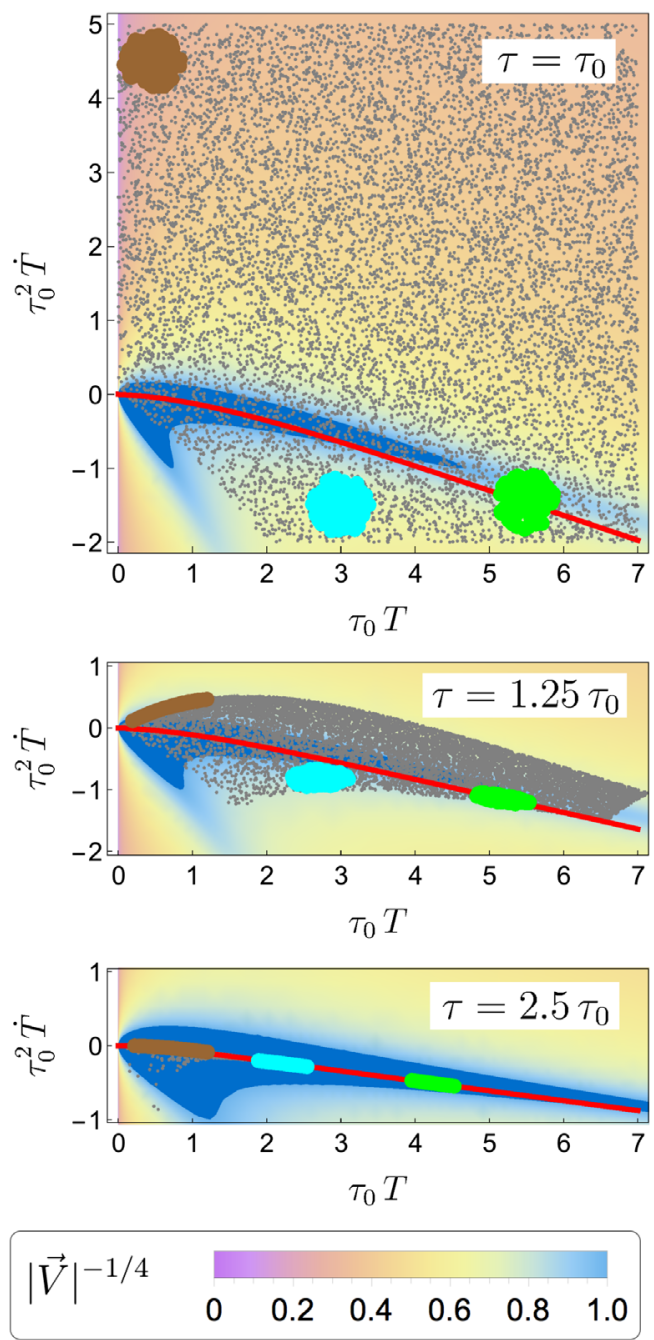

FIG. 1. Three snapshots of evolution in MIS-BRSSS phase space of a cloud of about 10000 random states in the region determined by the ranges of the top plot. The red curve denotes the family of solutions corresponding to the attractor $\mathcal{A}_{\star}(\tau T)$. The background color represents the speed at which the points move in phase space, with magenta being faster than blue according to the velocity defined in the text. The dark blue denotes the slow region beyond the color coding scale. For the purposes of visualizing local dimensionality reduction, see also Fig. 2, we track three initially spherical regions. The plots were made for $C_{\eta}=0.75$ and $C_{\tau_{\pi}}=1$, see Eqs. (3) and (4), and $\tau_{0}$ denotes initialization time.

Dimensionality reduction is signalled when one of the components is much smaller than the other one.

This analysis extends to phase spaces of arbitrary dimension. An interesting example is provided by the three-dimensional phase space of HJSW. The evolution of principal components is shown in the upper part of Fig. 3. There are three stages of dimensionality reduction. The first, from three to two dimensions, is analogous to the earliest phase of the collapse in MIS-BRSSS models, most likely due to the expansion. The second stage is 

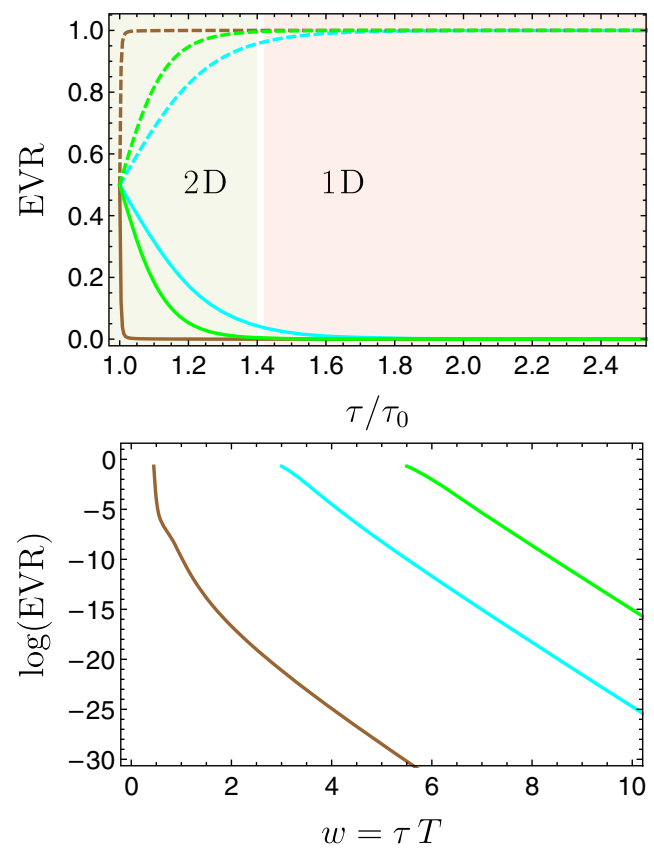

FIG. 2. Top: evolution of explained variance ratio of each principal component in MIS-BRSSS models for circles (radius: $10^{-4}$ ) of initial conditions with centres lying in the middle of initial dots of corresponding color in Fig. 1. Bottom: for large enough values of $w$ there is an exponential decay with a decay rate consistent with twice the transient mode contribution to $\mathcal{A}(w)$. The initial dimensional reduction of the brown region we view as triggered by the expansion rather than by nonhydrodynamic mode decay [22].

characterized by oscillations typical of the nonhydrodynamic sector of a HJSW model. These eventually dissipate resulting in the final stage of one-dimensional evolution.

Slow-roll in phase space.-The basic intuition is that the attractor locus should correspond to a region where the flow in phase space is slowest. One way to motivate why the slow region should behave as an attractor is to use an argument inspired by thermodynamics: a system is likely to be found in a large entropy macrostate because such states cover the majority of phase space. In our setting, the system is likely to be in a slow region because it takes a long time to escape it, while the fast regions can be quickly traversed.

We have found that, in the case of MIS-BRSSS models, this idea correctly identifies the attractor on any given time slice. Let $\vec{X}(\tau)=\left[\tau_{0} T(\tau), \tau_{0}^{2} \dot{T}(\tau)\right]$ be a point in a slice of phase space at time $\tau$ and $\tau_{0}$ denotes initialization time. This point moves with velocity $\vec{V}=\tau_{0} \vec{X}(\tau)$. The slow region is defined by its Euclidean norm $V$, which has a minimum at asymptotically late times when the system approaches local thermal equilibrium. However, the whole region where it is small is of dynamical significance. In Fig. 1, the background color is determined by $V$, where bluer color implies lower speed. There is a slow region stretching out from local thermal equilibrium, and the attractor $\mathcal{A}_{*}(w)$ lies

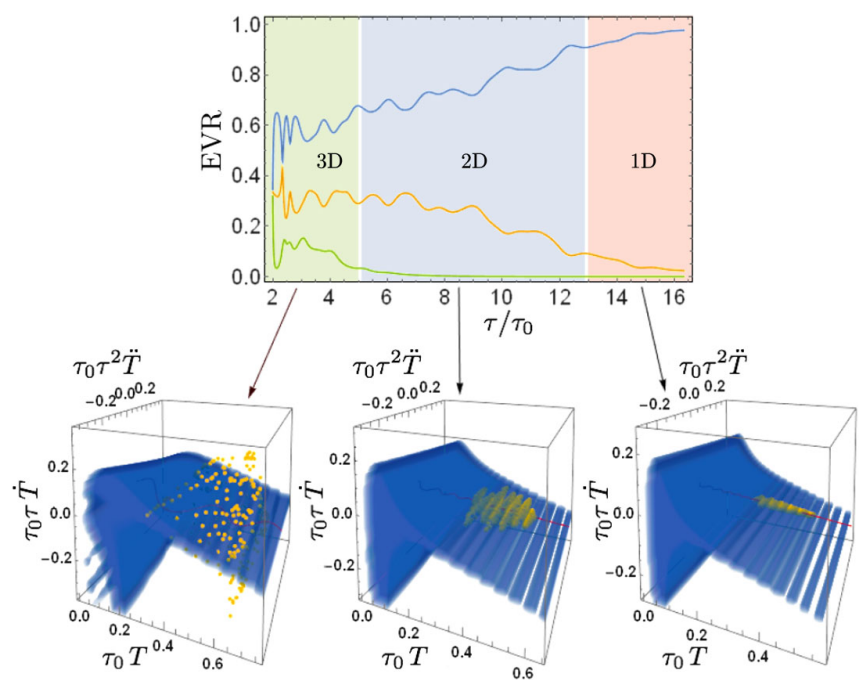

FIG. 3. In HJSW, the evolution of a cloud in phase space can be split into three stages, corresponding to the dimensionality of the cloud. The reduction from three to two dimensions corresponds to a collapse onto the slow region (blue region in plots). The plots were made with $C_{\eta}=0.75, C_{\tau_{\pi}}=1.16$, and $C_{\omega}=9.8$.

along it. It can be approximated by a slow-roll approximation [2] where one neglects $\ddot{T}$ in the equations of motion. This generalizes directly to phase spaces of any dimension. For the case of a HJSW model, the slow regions are shown also in blue in the bottom row of Fig. 3.

In nonautonomous systems, the slow region changes with time. If it were to evolve faster than the solutions do, it would not be useful to characterize the attractor. This is however not the case here: in both Figs. 1 and 3, we observe that once solutions reach the slow region, they stay inside and evolve with it. This is analogous to the adiabatic evolution observed in the case of the Boltzmann equation in the relaxation time approximation considered in Ref. [24].

Multidimensional phase spaces.-In realistic settings, one may not be able to consider the full phase space, but only some finite-dimensional, approximate representation thereof. As an example of such a procedure, we have studied a 16-dimensional subspace of the phase space of the Boltzmann kinetic equation in the relaxation time approximation $[64,65]$. This subspace is captured by taking the lowest 16 moments of the distribution function and solving the evolution equations as in Ref. [18,19]. We followed the evolution of a set of 240 initial conditions spanning a fivedimensional manifold embedded in the 16-dimensional phase. The results of an exploratory analysis of the resulting dataset using PCA are illustrated by Fig. 4 and described in more detail in the Supplemental Material [66]. This preliminary study supports the viability of the proposed approach in multidimensional phase spaces.

Summary and outlook.-The pressure anisotropy $\mathcal{A}(w)$ has been observed to exhibit universal behavior in various models, characterized by different authors in terms of concepts such as pullback or forward attractors or slow 


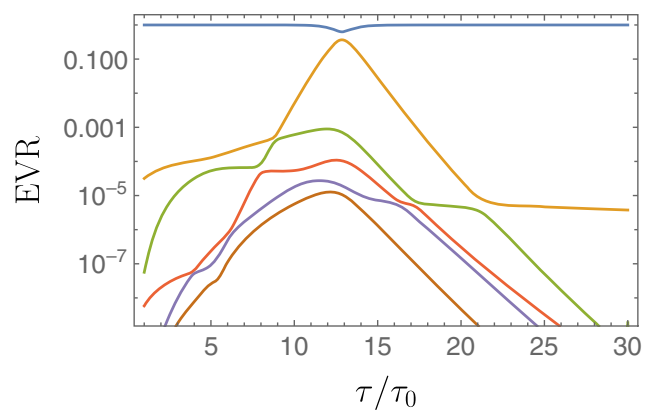

FIG. 4. The six largest principal components and their explained variance ratios as a function of $\tau$. Due to the bias in initial conditions, one component dominates already at the initial time. This direction is not dynamically preferred, as the other components initially grow in importance and only at $\tau \approx 15 \tau_{0}$ we see their expected exponential decay. At $\tau \approx 22 \tau_{0}$, the second principal component stabilizes. This feature may reflect the limitations of PCA to capture effects of curved regions in phase space.

roll, all of which capture some aspects information loss in dissipative systems. In this Letter, we have described hydrodynamic attractors using concepts that allow for generalizations to more realistic settings. We have shown that this can be achieved by considering the phase space of the theory, which alleviates the need to know in advance which particular quantity makes attractor behavior manifest. From this perspective, the hydrodynamic attractor is associated with the more general notion of dimensionality reduction of phase space regions.

We thank J. Carrasquilla, R. Janik, D. Teaney as well as participants of the XIV Polish Workshop on Relativistic Heavy-Ion Collisions at AGH, Initial Stages 2019 at Columbia University and Foundational Aspects of Relativistic Hydrodynamics at Banff International Research Station where this work was presented at different stages of development for helpful discussions. The Gravity, Quantum Fields and Information group at A.E. I. is supported by the Alexander von Humboldt Foundation and the Federal Ministry for Education and Research through the Sofja Kovalevskaja Award. M.S. was supported by the Polish National Science Centre Grant No. 2018/29/B/ST2/02457.

*michal.p.heller@aei.mpg.de †rjefferson@aei.mpg.de ${ }^{\ddagger}$ michal.spalinski@ncbj.gov.pl

§viktor.svensson@aei.mpg.de

[1] W. Busza, K. Rajagopal, and W. van der Schee, Heavy ion collisions: The big picture, and the big questions, Annu. Rev. Nucl. Part. Sci. 68, 339 (2018).

[2] M. P. Heller and M. Spaliński, Hydrodynamics Beyond the Gradient Expansion: Resurgence and Resummation, Phys. Rev. Lett. 115, 072501 (2015).
[3] G. Başar and G. V. Dunne, Hydrodynamics, resurgence, and transasymptotics, Phys. Rev. D 92, 125011 (2015).

[4] I. Aniceto and M. Spaliński, Resurgence in extended hydrodynamics, Phys. Rev. D 93, 085008 (2016).

[5] P. Romatschke, Relativistic Fluid Dynamics Far From Local Equilibrium, Phys. Rev. Lett. 120, 012301 (2018).

[6] M. Spaliński, On the hydrodynamic attractor of Yang-Mills plasma, Phys. Lett. B 776, 468 (2018).

[7] M. Strickland, J. Noronha, and G. Denicol, The anisotropic non-equilibrium hydrodynamic attractor, Phys. Rev. D 97, 036020 (2018).

[8] P. Romatschke, Relativistic hydrodynamic attractors with broken symmetries: Non-conformal and non-homogeneous, J. High Energy Phys. 12 (2017) 079.

[9] G. S. Denicol and J. Noronha, Analytical attractor and the divergence of the slow-roll expansion in relativistic hydrodynamics, Phys. Rev. D 97, 056021 (2018).

[10] W. Florkowski, E. Maksymiuk, and R. Ryblewski, Coupled kinetic equations for quarks and gluons in the relaxation time approximation, Phys. Rev. C 97, 024915 (2018).

[11] A. Behtash, C. N. Cruz-Camacho, and M. Martinez, Far-from-equilibrium attractors and nonlinear dynamical systems approach to the Gubser flow, Phys. Rev. D 97, 044041 (2018).

[12] J. Casalderrey-Solana, N. I. Gushterov, and B. Meiring, Resurgence and hydrodynamic attractors in Gauss-Bonnet holography, J. High Energy Phys. 04 (2018) 042.

[13] J.-P. Blaizot and L. Yan, Fluid dynamics of out of equilibrium boost invariant plasmas, Phys. Lett. B 780, 283 (2018).

[14] D. Almaalol and M. Strickland, Anisotropic hydrodynamics with a scalar collisional kernel, Phys. Rev. C 97, 044911 (2018).

[15] G. S. Denicol and J. Noronha, Hydrodynamic attractor and the fate of perturbative expansions in Gubser flow, Phys. Rev. D 99, 116004 (2019).

[16] A. Behtash, S. Kamata, M. Martinez, and C. N. Cruz-Camacho, Non-perturbative rheological behavior of a far-from-equilibrium expanding plasma, Phys. Lett. B 797, 134914 (2019).

[17] M. Spaliński, Universal behaviour, transients and attractors in supersymmetric Yang-Mills plasma, Phys. Lett. B 784, 21 (2018).

[18] M. Strickland, The non-equilibrium attractor for kinetic theory in relaxation time approximation, J. High Energy Phys. 12 (2018) 128.

[19] M. Strickland and U. Tantary, Exact solution for the nonequilibrium attractor in number-conserving relaxation time approximation, J. High Energy Phys. 10 (2019) 069.

[20] J.-P. Blaizot and L. Yan, Emergence of hydrodynamical behavior in expanding quark-gluon plasmas, Ann. Phys. (Amsterdam) 412, 167993 (2020).

[21] S. Jaiswal, C. Chattopadhyay, A. Jaiswal, S. Pal, and U. Heinz, Exact solutions and attractors of higher-order viscous fluid dynamics for Bjorken flow, Phys. Rev. C 100, 034901 (2019).

[22] A. Kurkela, W. van der Schee, U. A. Wiedemann, and B. Wu, Early- and Late-Time Behavior of Attractors in HeavyIon Collisions, Phys. Rev. Lett. 124, 102301 (2020). 
[23] G. S. Denicol and J. Noronha, Exact Hydrodynamic Attractor of an Ultrarelativistic Gas of Hard Spheres, Phys. Rev. Lett. 124, 152301 (2020).

[24] J. Brewer, L. Yan, and Y. Yin, Adiabatic hydrodynamization in rapidly-expanding quark-gluon plasma, arXiv:1910.00021.

[25] A. Behtash, S. Kamata, M. Martinez, and H. Shi, Global flow structure and exact formal transseries of the Gubser flow in kinetic theory, J. High Energy Phys. 07 (2020) 226.

[26] C. Chattopadhyay and U. W. Heinz, Hydrodynamics from free-streaming to thermalization and back again, Phys. Lett. B 801, 135158 (2020).

[27] A. Dash and V. Roy, Hydrodynamic attractors for Gubser flow, Phys. Lett. B 806, 135481 (2020).

[28] M. Shokri and F. Taghinavaz, Bjorken flow in the general frame and its attractor, Phys. Rev. D 102, 036022 (2020).

[29] D. Almaalol, A. Kurkela, and M. Strickland, following Letter, Non-Equilibrium Attractor in High-Temperature QCD Plasmas, Phys. Rev. Lett. 125, 122302 (2020).

[30] J.-P. Blaizot and L. Yan, Analytical attractor for Bjorken expansion, arXiv:2006.08815.

[31] T. Mitra, S. Mondkar, A. Mukhopadhyay, A. Rebhan, and A. Soloviev, Hydrodynamic attractor of a hybrid viscous fluid in Bjorken flow, arXiv:2006.09383.

[32] A. Mazeliauskas and J. Berges, Prescaling and Far-fromEquilibrium Hydrodynamics in the Quark-Gluon Plasma, Phys. Rev. Lett. 122, 122301 (2019).

[33] J. M. Maldacena, The large N limit of superconformal field theories and supergravity, Adv. Theor. Math. Phys. 2, 231 (1998).

[34] E. Witten, Anti-de Sitter space and holography, Adv. Theor. Math. Phys. 2, 253 (1998).

[35] S. Gubser, I. R. Klebanov, and A. M. Polyakov, Gauge theory correlators from noncritical string theory, Phys. Lett. B 428, 105 (1998).

[36] P. M. Chesler and W. van der Schee, Early thermalization, hydrodynamics and energy loss in AdS/CFT, Int. J. Mod. Phys. E 24, 1530011 (2015).

[37] J. Berges, M. P. Heller, A. Mazeliauskas, and R. Venugopalan, Thermalization in QCD: Theoretical approaches, phenomenological applications, and interdisciplinary connections, arXiv:2005.12299.

[38] P. B. Arnold, G. D. Moore, and L. G. Yaffe, Effective kinetic theory for high temperature gauge theories, J. High Energy Phys. 01 (2003) 030.

[39] S. Schlichting and D. Teaney, The first fm/c of heavy-ion collisions, Annu. Rev. Nucl. Part. Sci. 69, 447 (2019).

[40] I. Muller, Zum paradoxon der warmeleitungstheorie, Z. Phys. 198, 329 (1967).

[41] W. Israel and J. Stewart, Transient relativistic thermodynamics and kinetic theory, Ann. Phys. (N.Y.) 118, 341 (1979).

[42] R. Baier, P. Romatschke, D. T. Son, A. O. Starinets, and M. A. Stephanov, Relativistic viscous hydrodynamics, conformal invariance, and holography, J. High Energy Phys. 04 (2008) 100.

[43] A. R. Liddle, P. Parsons, and J. D. Barrow, Formalizing the slow roll approximation in inflation, Phys. Rev. D 50, 7222 (1994).

[44] M. Spalinski, On the slow roll expansion for brane inflation, J. Cosmol. Astropart. Phys. 04 (2007) 018.
[45] G. Giacalone, A. Mazeliauskas, and S. Schlichting, Hydrodynamic attractors, initial state energy and particle production in relativistic nuclear collisions, Phys. Rev. Lett. 123, 262301 (2019).

[46] T. Dore, E. McLaughlin, and J. Noronha-Hostler, Far from equilibrium hydrodynamics and the beam energy scan, J. Phys. Conf. Ser. 1602, 012017 (2020).

[47] A. Behtash, S. Kamata, M. Martinez, and H. Shi, Global flow structure and exact formal transseries of the gubser flow in kinetic theory, J. High Energy Phys. 07 (2020) 226.

[48] J. Bjorken, Highly relativistic nucleus-nucleus collisions: The central rapidity region, Phys. Rev. D 27, 140 (1983).

[49] R. A. Janik and R. B. Peschanski, Gauge/gravity duality and thermalization of a boost-invariant perfect fluid, Phys. Rev. D 74, 046007 (2006).

[50] M. P. Heller, R. A. Janik, and P. Witaszczyk, Hydrodynamic Gradient Expansion in Gauge Theory Plasmas, Phys. Rev. Lett. 110, 211602 (2013).

[51] I. Aniceto, B. Meiring, J. Jankowski, and M. Spaliński, The large proper-time expansion of Yang-Mills plasma as a resurgent transseries, J. High Energy Phys. 02 (2019) 073.

[52] I. Aniceto, G. Başar, and R. Schiappa, A primer on resurgent transseries and their asymptotics, Phys. Rep. 809, 1 (2019).

[53] W. Florkowski, M. P. Heller, and M. Spaliński, New theories of relativistic hydrodynamics in the LHC era, Rep. Prog. Phys. 81, 046001 (2018).

[54] R. Loganayagam, Entropy current in conformal hydrodynamics, J. High Energy Phys. 05 (2008) 087.

[55] M. P. Heller, R. A. Janik, M. Spaliński, and P. Witaszczyk, Coupling Hydrodynamics to Nonequilibrium Degrees of Freedom in Strongly Interacting Quark-Gluon Plasma, Phys. Rev. Lett. 113, 261601 (2014).

[56] J. Noronha and G. S. Denicol, Transient fluid dynamics of the quark-gluon plasma according to AdS/CFT, arXiv:1104.2415.

[57] P. K. Kovtun and A. O. Starinets, Quasinormal modes and holography, Phys. Rev. D 72, 086009 (2005).

[58] P. E. Kloeden and M. Rasmussen, Nonautonomous Dynamical Systems (American Mathematical Society, Providence, 2011), Vol. 176.

[59] R. S. Bhalerao, J.-Y. Ollitrault, S. Pal, and D. Teaney, Principal Component Analysis of Event-By-Event Fluctuations, Phys. Rev. Lett. 114, 152301 (2015).

[60] A. Mazeliauskas and D. Teaney, Fluctuations of harmonic and radial flow in heavy ion collisions with principal components, Phys. Rev. C 93, 024913 (2016).

[61] P. Bozek, Principal component analysis of the nonlinear coupling of harmonic modes in heavy-ion collisions, Phys. Rev. C 97, 034905 (2018).

[62] Z. Liu, W. Zhao, and H. Song, Principal component analysis of collective flow in relativistic heavy-ion collisions, Eur. Phys. J. C 79, 870 (2019).

[63] F. G. Gardim, F. Grassi, P. Ishida, M. Luzum, and J.-Y. Ollitrault, $p_{T}$-dependent particle number fluctuations from principal component analyses in hydrodynamic simulations of heavy-ion collisions, Phys. Rev. C 100, 054905 (2019).

[64] G. Baym, Thermal Equilibration in Ultrarelativistic Heavy Ion Collisions, Phys. Lett. B 138, 18 (1984). 
[65] W. Florkowski, R. Ryblewski, and M. Strickland, Testing viscous and anisotropic hydrodynamics in an exactly solvable case, Phys. Rev. C 88, 024903 (2013).

[66] See the Supplemental Material at http://link.aps.org/ supplemental/10.1103/PhysRevLett.125.132301 for more details on dimensionality reduction in kinetic theory, which includes Refs. [67,68].
[67] M. P. Heller, A. Kurkela, M. Spaliński, and V. Svensson, Hydrodynamization in kinetic theory: Transient modes and the gradient expansion, Phys. Rev. D 97, 091503 (2018).

[68] M. P. Heller and V. Svensson, How does relativistic kinetic theory remember about initial conditions?, Phys. Rev. D 98, 054016 (2018). 